\section{Fernsehen oder Videospiel - die Aufmerksamkeit leidet ...}

\begin{abstract}
Die Tatsache, dass Fernsehen Aufmerksamkeitsprobleme verursacht, ist nicht neu. Eine amerikanische Arbeitsgruppe hat sich in einer Längsschnittuntersuchung der Frage zugewandt, ob dies auch für Video- und PC-Spiele gilt.
\end{abstract}

— ür die Untersuchung wurden 1.323 Kinder im Alter von sechs bis zwölf

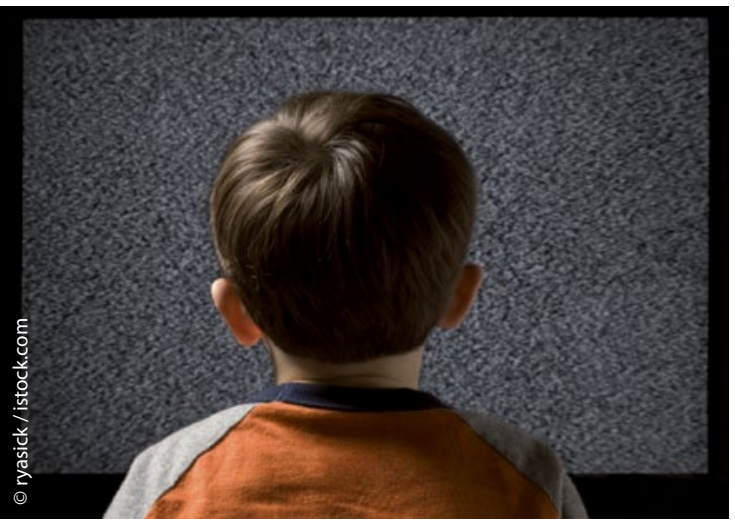

Jahren in einer 13 Monate währenden Periode beobachtet. Dabei registrierten die Eltern und die Kinder die Zeiten vor dem Fernseher und beim Spielen vor dem Bildschirm. Die Lehrer der Kinder sollten schließlich mithilfe von jeweils drei Merkmalen in einer fünfstufigen Skala die Aufmerksamkeitsprobleme bewerten.

Als Ergebnis zeigte sich, dass die Nutzung von Bildschirmgeräten unabhängig davon, ob es sich um Fernsehen oder Videospiele handelt, die Aufmerksamkeit negativ beeinflusst. Die Auswirkung war bei Jungen stärker als bei Mädchen. Der Effekt war auch in der Gruppe junger Erwachsener $(n=210)$ nachweisbar.

\section{... oder doch nicht?}

Eine weitere Studie befasst sich mit der Frage, welche Faktoren Aufmerksamkeits- und Schulprobleme hervorrufen. Der Autor kommt zu einem anderen Schluss als Swing und Kollegen in der oben aufgeführten Studie.

E ür ür die Untersuchung wurde bei 603 Kinder im Alter zwischen zehn und 14 Jahren u. a. der Schulerfolg gemessen, demographische Daten erfasst, der visuelle Medienkonsum erhoben sowie die Child Behaviour Checklist (CBCL) ausgefüllt und das "negativ life events“ (NLE)-Instrument eingesetzt.

Der Autor kommt mithilfe von bivariaten Analysen der Daten zum Schluss, dass Faktoren wie Geschlecht, antisoziales Verhalten, familiäres Umfeld und Ängstlichkeit am stärksten mit Aufmerksamkeitsproblemen korrelierten. Der Schulerfolg hingegen stand vor allem mit dem familiären Einkommen in Verbindung. Der visuelle Medienkonsum beeinflusste hingegen weder die Aufmerksamkeit noch die Schulleistungen.

Kommentar: Der Einfluss von visuellem Medienkonsum wird kontrovers diskutiert. Während Christiakis et al. (2004) einen negativen Einfluss beobachteten, konnte
Stevens et al. (2006) dies nicht bestätigen. Swing et al. (2010) fanden in der oben besprochenen Studie einen leichten negativen Einfluss. Bei Spence et al. (2010) kam hingegen ein positiver Effekt auf die Kognition durch Videospiele heraus. Diese divergierenden Befunde machen deutlich, wie interpretiert wird. So stellt sich immer wieder die Frage, ob Aufmerksamkeits- und Schulprobleme zu erhöhtem visuellen $\mathrm{Me}$ dienkonsum führen oder umgekehrt. Oder ob ein weiterer Faktor, z. B. das familiäre Umfeld, sowohl die Aufmerksamkeits- und Schulprobleme als auch den visuellen Medienkonsum beeinflusst.

Ferguson konnte in der vorliegenden Studie hingegen keine Korrelation zwischen visuellem Medienkonsum und diesen Faktoren beobachten. Hier ist jedoch kritisch zu bemerken, dass nur die Kinder selbst nach der Art und Dauer ihres Medienkonsums befragt wurden. Aus der praktischen Erfahrung ist aber bekannt, wichtig es ist, was und wie untersucht bzw.
Kommentar: In Deutschland dürften diese Ergebnisse niemanden überraschen. Hierzulande ist die Datenbasis durch die Untersuchungen des Hannoveraner Kriminologen Christian Pfeiffer wesentlich breiter als die in der Studie von Swing und Kollegen. In den 35 Literaturangaben zu deren Arbeit werden fast ausschließlich Untersuchungen von Ärzten, wenige von Psychologen und gar keine von Kriminologen zitiert. Pfeiffer hat in Deutschland wesentlich eindrucksvollere Daten vorgelegt [www.kfn.de/versions/kfn/ assets/zjj.pdf]. Er hat es auf den Punkt gebracht mit dem Nachweis, dass uns in Deutschland jedes Jahr durch Bildschirmgeräte in Kinderzimmern 30.000 männliche Abiturienten entgehen. Das dürfte sich das Land kaum leisten können.

Dr. Hartmut Koch

Swing EL et al. Television and video game exposure and the development of attention problems. Pediatrics 2010; 126: 214-21

dass gerade Kinder und Teenager diesen in der Regel zu niedrig angeben.

Die höchste Korrelation zu Aufmerksamkeitsproblemen bestand mit Angststörungen (gemessen anhand des CBCL). Dieser Zusammenhang wurde auch schon von anderen Autoren bestätigt. Hier stellt sich aber erneut die Frage, wie die Beziehung zwischen Angst und Aufmerksamkeitsstörung aussieht: Führt die Angstsymptomatik zur Aufmerksamkeitsstörung - oder umgekehrt? Führen durch die Aufmerksamkeitsstörung entstandene Misserfolge zu Minderung des Selbstwertgefühles, zu vermehrter Unsicherheit und einem ängstlichen Verhalten?

Die Ergebnisse der vorliegenden Arbeit werfen mehr Fragen auf als sie beantworten. Es bleibt zu hoffen, dass Einflüsse des Medienkonsums auf das Verhalten und die Lernfähigkeit des Kindes weiter beforscht werden. Hier sollte auch mehr differenziert werden, ob der Konsum am Stück oder in mehrerer Häppchen stattfindet und was konsumiert wird.

\section{Dr. Kirsten Stollhoff}

Ferguson CJ. The influence of television and video game use on attention and school problems. J Psychiatr Res 2011; 45: 808-13 